DOI 10.18699/PlantGen2019-126

\title{
Association mapping for physio-biochemical traits \\ under salt stress in wheat RILs population developed from cross between Frontana $\times$ Pasban 90
}

\author{
Noshin I.*, Naziam B., Faisal Q. \\ Department of Botany, PMAS Arid Agriculture University, Rawalpindi, Pakistan \\ *e-mail:noshinilyas@yahho.com; armghan_shehzad@yahoo.com
}

In present study a population of recombinant in hybrid lines develop from cross between Frontana and Pasban90 parents was evaluated for (5) morphological, (7) physiological and (6) biochemical traits under normal and salt stress environment in hydroponic culture for two cropping season 2014-15 and 2015-16. Parents and their RILs showed considerable variation in studied traits. Total 202 polymorphic microsatellite markers (SSR) and composite interval mapping approach was used. Present study identified ninety-two significant QTLs out of which 27 were major and 65 were minor and most were concentrated on chromosome 2A, 2B and 7A (7 each). Twelve major QTLs were reported on $\mathrm{B}$ genome followed by $\mathrm{D}$ and $\mathrm{A}$ while chromosome $4 \mathrm{~B}$ had maximum number of major QTLs. QTLs for sodium and potassium content were concentrated on D genome due to presence of Knal gene on D genome. Furthermore, thirty-two pleiotropic regions were identified and chromosome $2 \mathrm{~A}$ contained pleotropic region associated with five different traits. The identified MTAs may be helpful in improving wheat tolerance to salt and can be employed in marker-assisted selection. 